\title{
Vom Fuße des Himalaya - Wertschöpfungskette Äpfel in Indien
}

\author{
Sabine Golombek ${ }^{1} \cdot$ Michael Blanke $^{1}$ \\ Eingegangen: 15. Mai 2019 / Angenommen: 26. Juni 2020 / Online publiziert: 5. August 2020 \\ (c) Der/die Autor(en) 2020, korrigierte Publikation 2020
}

\section{Zusammenfassung}

Trotz eines geringen Apfelkonsums von nur ca. 2 kg/Kopf/Jahr ist Indien mit ca. 2,3 Mio.t (2017) Äpfeln - auf $314.000 \mathrm{ha}-$ nach China, USA, Polen und der Türkei - der fünftgrößte Apfelproduzent der Welt; dabei macht der Apfelanbau nur 2,4\% der gesamten Obstproduktion des Landes aus und konzentriert sich auf die 4 nordwestlichen Provinzen Jammu und Kaschmir (ca. 70\%), Himachal Pradesh (ca. 20\%), Uttarakhand (ca. 10\%) und Arunachal Pradesh, wo in Höhen von 1000-3500m am Fuße des Himalaya mit 800-1600 Kältestunden (CH - Chilling hours) ausreichend Kältereiz für die Blütenbildung zur Verfügung steht. Apfel ist für Kleinbauern - im Vergleich zu Gemüse - eine lukrative Kultur, trotz geringer Ernteerträge auf Hanglagen von durchschnittlich 10t/ha aus der 'Red Delicious'-Gruppe, ursprünglich auf Sämlingsunterlage.

Erhebliche Verluste entstehen durch unsachgemäße Ernte, Mangel an Obstlagern und für die langen Entfernungen und schlechten Straßen häufig unzureichende (plastikfreie) Verpackung aus Stroh und Zeitungspapier in Kartons oder hohen harten Holzkisten und Transport ohne Kühlung.

Exportiert werden Mangos, Tafeltrauben und Granatäpfel und ca. 18.000 t Äpfel im Wert von 9 Mio. US \$ in das angrenzende Bangladesh und Nepal. Ein Importembargo für Äpfel aus China seit Mai 2017, offiziell wegen Schädlingsbefall, reduzierte die Apfelimporte von 370.000t (2016/17) auf 250.000t in 2017/18 und 270.000t in 2018/19. Importiert werden Äpfel der Sorten 'Fuji', 'Red Delicious', 'Gala', 'Granny Smith' u. a. aus US, (China), Neuseeland, Chile und Südtirol; bisher stammte die Hälfte der Apfelimporte aus Washington State, deren Einfuhrzoll nach Wirtschaftssanktionen von 50 auf $75 \%$ angehoben wurde.

Der indische Markt bzw. Konsument bevorzugt große, rote (oder grüne) Äpfel mit geringen Schalenfehlern. Die hohen Großhandelspreise von 28-35Rs/kg (0,34-0,43€/kg) für heimische und 56-145Rs/kg (0,69-1,77€/kg) für Importäpfel im Januar 2019 bieten Apfelexporteuren ein Marktpotential von ca. 200Mio. $€$ jährlich. Hohe Preise und Handarbeit dominieren in der Apfel-Wertschöpfungskette, die so auch vielen ungelernten Menschen ein Einkommen sichert.

Schlüsselwörter Apfel (Malus domestica Borkh) · Indien · Kleinbauern · Klimawandel · Lebensmittelverluste · Nachhaltigkeit · Transportverluste · Plastikfreie Verpackung · Wertschöpfungskette

Michael Blanke

mmblanke@uni-bonn.de

1 INRES-Gartenbauwissenschaft, Universität Bonn, Auf dem Hügel 6, 53121 Bonn, Deutschland 


\title{
Apple Supply Chain in India-From The Himalayan Mountains to The Consumer
}

\begin{abstract}
With nearly 2.3 mil t apple cultivation on 314,000 ha, India ranks as the 5th largest apple producer worldwide, but amounts to only $2.4 \%$ of India's overall fruit production. Apple cultivation is traditionally concentrated in the provinces Jammu and Kaschmir (ca. 70\%), Himachal Pradesh (ca. 20\%), Uttarakhand (ca. 10\%) und Arunachal Pradesh in the foothills (1000 to $3500 \mathrm{~m}$ ) of the Himalayan mountains with $800-1600 \mathrm{CH}$ provides sufficient chilling. Despite low yields of an averaged $10 t / h a$, apple of the 'Red Delicious' group is an economic crop-relative to vegetables-for smallholder farmers. The fruits are transported 4 to 8 days per truck sometimes on poor roads and without cooling to their destination, whereas imported fruit arriving at a port benefit from shorter inland transport and cool chain. Lack of harvest techniques, storage facilities, refrigerated transport and protective packaging results in considerable losses along the domestic apple supply chain.

The Indian apple market with an estimated apple consumption of ca. 3.1 milt/year or ca. $2 \mathrm{~kg} / \mathrm{head}$ per year is supplied ca. $90 \%$ (2-3 milt) by inland production and by ca. $10 \%$ by apple imports. Imports dropped from 370,000t in 2016/7 (worth US \$200,000) to 250,000t in 2017/8 and 270,000t in 2018/19 after an import ban for apples from China due alleged sanitary contamination from May 2017 and increase in import duty on US fruit from 50 to $75 \%$. International fruit suppliers are the US, (China), New Zealand, Chile and Italy with apple cvs 'Fuji', 'Red Delicious', 'Gala', 'Granny Smith', etc. with an overall market potential of ca. 200 mil US \$. India exports mangos, table grapes and pomegranates, as well as ca. 18,000t apples (worth 9 mil US \$) to neighbouring Bangladesh and Nepal.

The Indian market and consumer prefers large, red (or green) essentially blemish-free apples, which fetch high prices from $28-35 \mathrm{Rs} / \mathrm{kg}(0.34-0.43 € / \mathrm{kg})$ for domestic to $56-145 \mathrm{Rs} / \mathrm{kg}(0.69-1.77 € / \mathrm{kg} ;$ Jan 2019) for imported fruit, the latter with 200 mil euros attractive for the international apple trade. (Un-)loading, grading, transport and fruit stalls are dominated by manual labour and offer unskilled people social security and income.
\end{abstract}

Keywords Apple (Malus domestica Borkh) $\cdot$ Climate change $\cdot$ Food waste $\cdot$ India $\cdot$ Plastic free packaging $\cdot$ Smallholder farmer $\cdot$ Supply chain $\cdot$ Sustainability

\section{Einleitung}

Auf der INTERPOMA 2018 in Bozen wurde Indien als das Land mit dem größten Potential für Äpfel identifiziert (Arora 2018). Auch wenn China mit seinen 44 Mio.t Äpfel in 2016 (FAO 2018) mit Abstand weltweit die Spitzenposition in der Apfelproduktion einnimmt, gilt Indien als Hoffnungsträger Nr. 1 sowohl im Apfelkonsum als auch in der Apfelproduktion. Ein Anstieg der indischen Apfelnachfrage bzw. -konsums um $500 \mathrm{~g} / \mathrm{Kopf}$, also nur 3 Äpfel zusätzlich pro Kopf und Jahr, z. B. durch zunehmenden Wohlstand und Gesundheitsbewusstsein, bedeutet eine Nachfragesteigerung um 700.000t (Arora 2018), die der inländische Anbau im Moment noch nicht befriedigen kann, aber das Potential dazu hätte. Indien ist für Washington State der drittgrößte und wichtigste Apfelabnehmer, vor allem, seit ab Mai 2017 in Indien ein Embargo bzw. Importverbot für chinesische Äpfel besteht. Dieser Beitrag schildert persönliche Eindrücke von Studienreisen der Autorin - zuletzt im Januar 2019 - zur Apfel Supply Chain/Wertschöpfungskette in Indien.

\section{Lage der Obstanbaugebiete - Chilling (Kältereiz) als begrenzender Anbaufaktor und Ursprung der Apfelsorten}

Indien mit seinen 1,3 Mrd. Einwohnern besitzt eine im Vergleich zur Weltbevölkerung inzwischen nur noch durchschnittlich wachsende Bevölkerung (1,1\% pro Jahr) mit (langsam) steigendem Wohlstand (Weltbank 2018). Das Bruttoinlandsprodukt beträgt nur US \$1800/Person/Jahr - bei ungleicher Verteilung und einem jährlichen Anstieg von 7,3\% (2017) im Vergleich zu China mit US \$ 8690/ Person/Jahr bei ähnlichem Bevölkerungswachstum und Einwohnerzahl. Der heimische Apfelanbau konzentriert sich auf die 4 nordwestlichen Provinzen (Abb. 1) Jammu und Kaschmir (ca. 70\%), Himachal Pradesh (ca. 20\%), Uttarakhand (ca. 10\%) und Arunachal Pradesh (Abb. 1) (Horticulture Statistics Division 2017). Der Obstbau erfolgt in Höhen von 1000-3500m am Fuße des Himalaya: Nur dort stehen mit 800-1600 CH (Chilling hours) (Chauhan 2016) (Tab. 1) ausreichend Kältereiz (Chilling) für die Blütenbildung zur Verfügung. Wie bei uns wandern die Obstanbaugebiete im Himalaya mit dem Klimawandel nach Norden und von den Tal- in die Höhenlagen (Singh und Patel 2017). Mit den englischen Kolonialherren kamen um 1870 die ersten Apfelbäume mit typisch englischen 


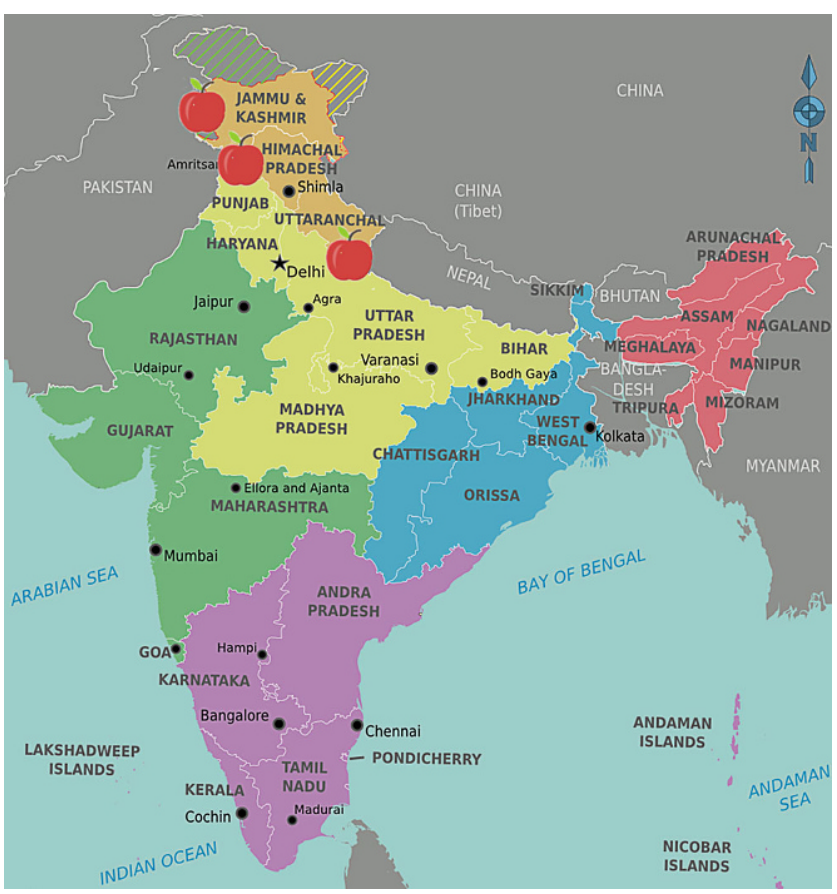

Abb. 1 Konzentration der Apfelanbaugebiete im Nordwesten Indiens am Fuße des Himalaya-Gebirges (Die Landkarte von Ravikiran Rao und Nichalp steht in einer Open Source Lizenz zur Verfügung: https:// wikitravel.org/shared/File:Map_of_India.svg und wurde verändert)

Sorten, Sämlinge und dem großen, grünen triploiden Kochapfel 'Bramley', in diese Region, bis der dort ansässige Samuel Evan Stokes 1918 amerikanische 'Red Delicious" Mutanten (Tab. 1) wie „Red Chief“", „Jeromine“, u. a. mit 'Gala' als Befruchter aus seiner Heimat mitbrachte, die heute den Anbau dominieren. So stieg die Anbaufläche in H. Pradesh von 400 ha in 1950 auf heute ca. 100.000 ha mit zunehmender Tendenz und stark wachsende Sämlinge werden langsam durch schwächer wachsende Unterlagen ersetzt.

Seit einigen Jahren wird an Grenzstandorten südlich dieser traditionellen Anbaugebiete im Himalaya in höher gelegenen Regionen in Südindien mit dem lukrativen Apfelanbau und Sorten mit geringem Kälteanspruch geforscht. Diese Experimente werden vom indischen Staat mit wissenschaftlicher Begleitung gefördert oder in Privatinitiative durchgeführt, mit dem Ziel der Einkommensverbesserung und der Versorgung lokaler Märkte mit frischen, einheimischen Äpfeln.

\section{Farmgröße, Eigentumsverhältnisse und Erträge - hohe Preise für Premiumäpfel}

Die typische Betriebsfläche in Himachal Pradesh beträgt ca. 1,2 ha (3,1 acres) und ernährt eine Familie. Das Land gehört den Kleinbauern und wird weiter vererbt - eine gute Voraussetzung für nachhaltigen Anbau (Terlau et al. 2018). Apfel ist für die Landwirte die produktivste Kultur - im Vergleich zu Gemüse. Trotz offizieller Maut-Befreiung von Obst/Gemüse müssen Transportgebühren und eine Verkaufskommission von ca. 5-6\% gezahlt werden. Die durchschnittliche Erzeugung des Landes liegt von ca. 2,3 Mio. t Äpfel auf über 300.000 ha (Abb. 2) - im Vergleich zu Deutschland mit 900.000t auf 33.000 ha -, d.h. ca. 10t/ha, wobei die Erträge pro Baum in Kleinstparzellen in den Hanglagen Nordindiens schwierig auf ha umzurechnen sind.

\section{Anbau und Apfel Obstexporte Indiens - Größtes Importpotential für Äpfel weltweit}

Die Apfelernte erfolgt - je nach Höhenlage - von Ende Juli/August bis November zur gleichen Zeit wie bei uns in Europa, in den USA oder in China. Indien ist mit 1,89Mio. $t$ (2013), 2,2 (2015) bis 2,89 Mio. t (2017) und 2,2-2,3 Mio. t (2018) Äpfel (FAO 2018; Abb. 2) auf 314.000 ha - nach China, USA, Polen und der Türkei - der fünftgrößte Apfelproduzent der Welt. Die geerntete Apfelmenge entspricht etwa der Größenordnung Italiens, Irans oder der Türkei. Trotz der beeindruckenden Zahlen macht die Apfelproduktion nur 2,4\% der gesamten Obstproduktion Indiens aus (Arora 2018).

Indien ist regelmäßig mit einem Gemeinschaftstand auf der Fruit Logistica in Berlin vertreten (Abb. 3). Exportiert werden Mangos, Tafeltrauben - nach England, Holland und Deutschland (Abb. 4) (Golombek und Blanke 2020) und Granatäpfel, aber auch ca. 18.000t Äpfel im Wert von 9 Mio. US \$ ins benachbarte Bangladesh und Nepal (Arora 2018) - die Apfelimporte übersteigen diesen Wert um den Faktor 24

Die Apfelimporte liegen zwischen 190.000t (2014/15), 240.000t (2015/16), bis 370.000 (2016/17) (FAO 2018; Werth und Kager 2018) und nach dem Chinabann wiederum bei nur 250.000t (2017/18) und 270.000t (2018/19) (Ministry of Commerce und Industry 2019). Damit bietet Indien den Apfelexporteuren ein enormes Marktpotential von

Tab. 1 Typischer Apfelanbau im Nordwesten Indiens

\begin{tabular}{llll}
\hline Parameter & Mittelwert & Parameter & Sorten und Erträge \\
\hline Regenmenge & $1430 \mathrm{~mm} / \mathrm{Jahr}$ & Sorten & 'Red Delicious' Gruppe \\
Kältereiz & $1200 \mathrm{CH} / \mathrm{Jahr}$ & Unterlage & Bisher Sämling \\
Farmgröße & $1,2 \mathrm{ha}$ & Ertrag & $3-10 \mathrm{t} / \mathrm{ha} \mathrm{u}$. Jahr \\
\hline
\end{tabular}


Abb. 2 Apfelproduktion und Apfelimporte Indiens seit 2013

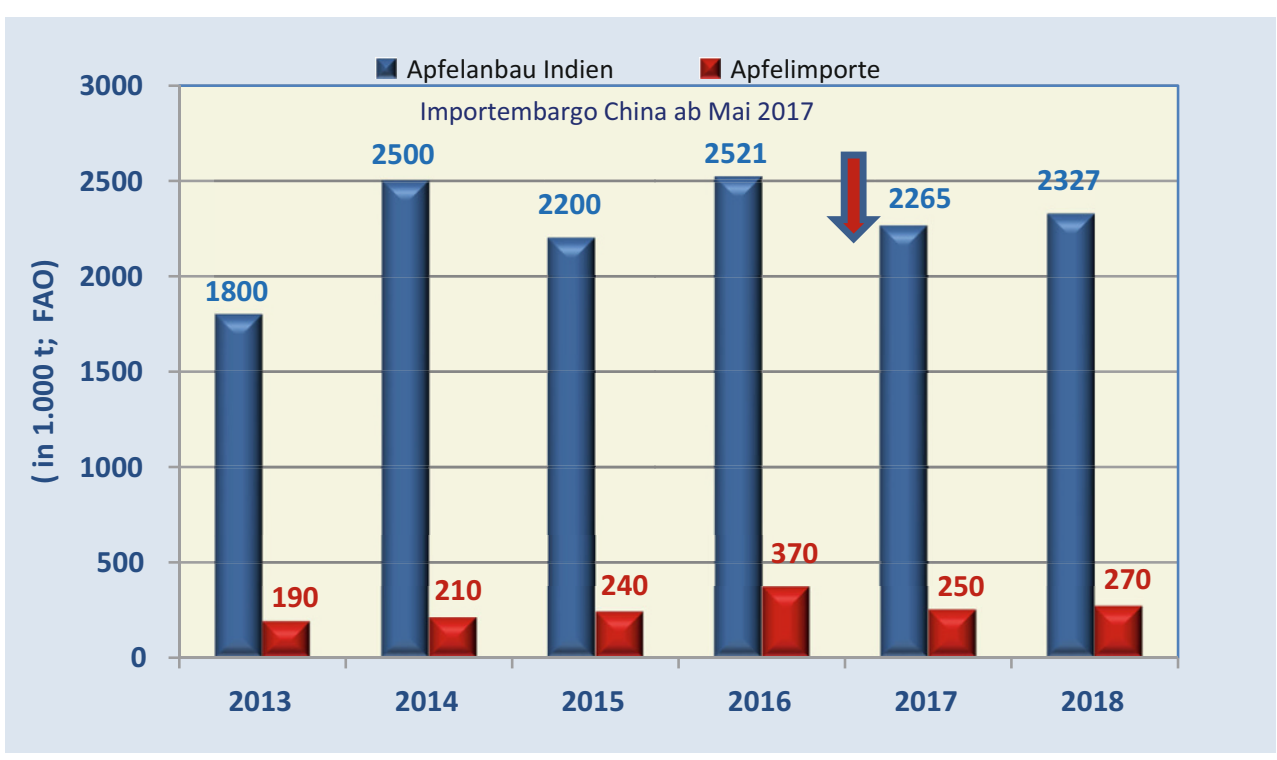

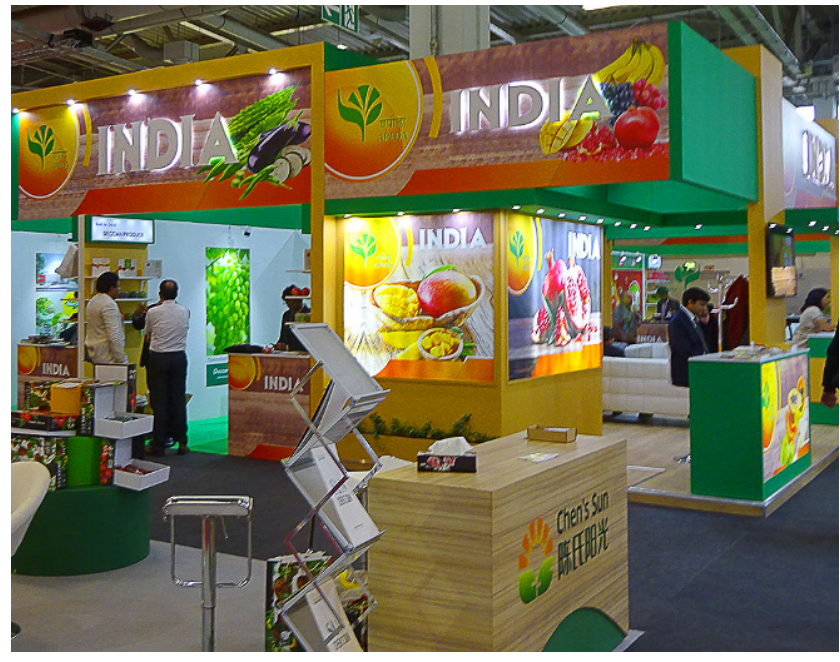

Abb. 3 Indiens Gemeinschaftsstand auf der Fruit Logistica in Berlin 2019

ca. 200 Mio. $€$ jährlich unter strengen Importbestimmungen. Fast die Hälfte der Apfelimporte stammte bisher aus Washington State, von dort sind die Schiffe ca. 40 Tage, von Europa nur ca. 20 Tage unterwegs, sodass Europa einen Handelsvorteil hätte (Arora 2018), aber nur einen Anteil von ca. $10 \%$ an den Apfelimporten hat, davon Italien etwa die Hälfte; Lieferländer der Südhalbkugel sind Chile und Neuseeland (Abb. 5); von Deutschland wurde Anfang 2019 eine erste Ladung 'Jonagold'-Äpfel versuchsweise nach Indien verschifft.

\section{Importembargo für Äpfel aus China und hohe Einfuhrzölle für USA}

Das vorübergehende Importembargo für Äpfel aus China seit Mai 2017 wurde offiziell wegen wiederholten Auftretens der asiatischen Schildlaus Pseudococcus comstocki und des Pilzes Fusarium oxysporum bei Einfuhrkontrollen verhängt. Apfelimporteure müssen bei der Einfuhr ein phytosanitäres Zertifikat, z. B. eine Desinfektion mit Methylbromid, vorweisen und Zoll bezahlen.

\section{Erhebliche Verluste von der Ernte bis zur Lagerung}

Mögliche Ursachen für Druckstellen, Verletzungen und Fäulnis der Früchte (Khan et al. 2018) reichen von unsanfter Ernte, Ausschütten der Erntekörbe, unsachgemäßer Sortierung bis zum Langstreckentransport. Aus den Hanglagen müssen die Äpfel erst von Familienmitgliedern, Trägern oder Eseln zu den nächsten Straßen getragen werden (Kireeti und Sharma 2017) bevor sie in 10 oder $20 \mathrm{~kg}$ Pappkartons oder $20 \mathrm{~kg}$ Holzkisten gepackt werden; Zwischenlagen bestehen aus Zeitung - Stroh - Zeitung und Streifen aus Zeitungspapier um die einzelnen Früchte. Die Verpackung ist plastikfrei, entsprechend einer staatlichen Verordnung zur Vermeidung von Einwegplastik vom 1. Januar 2019. Der Abtransport der Früchte geschieht oft ungekühlt (Abb. 6) 4 bis 8 Tage zu den entfernten Obstgroßmärkten z. T. über schlechte Straßen oder über bessere Straßen und dann mit Maut, die für Obst und Gemüse offiziell erlassen wurde. Bei warmem Wetter können die Verluste 2-3 kg pro 20-kg-Box, d.h. 10-15\%, ausmachen. Bei der Ankunft am Zielort werden Äpfel z.B. mit Druckstellen 

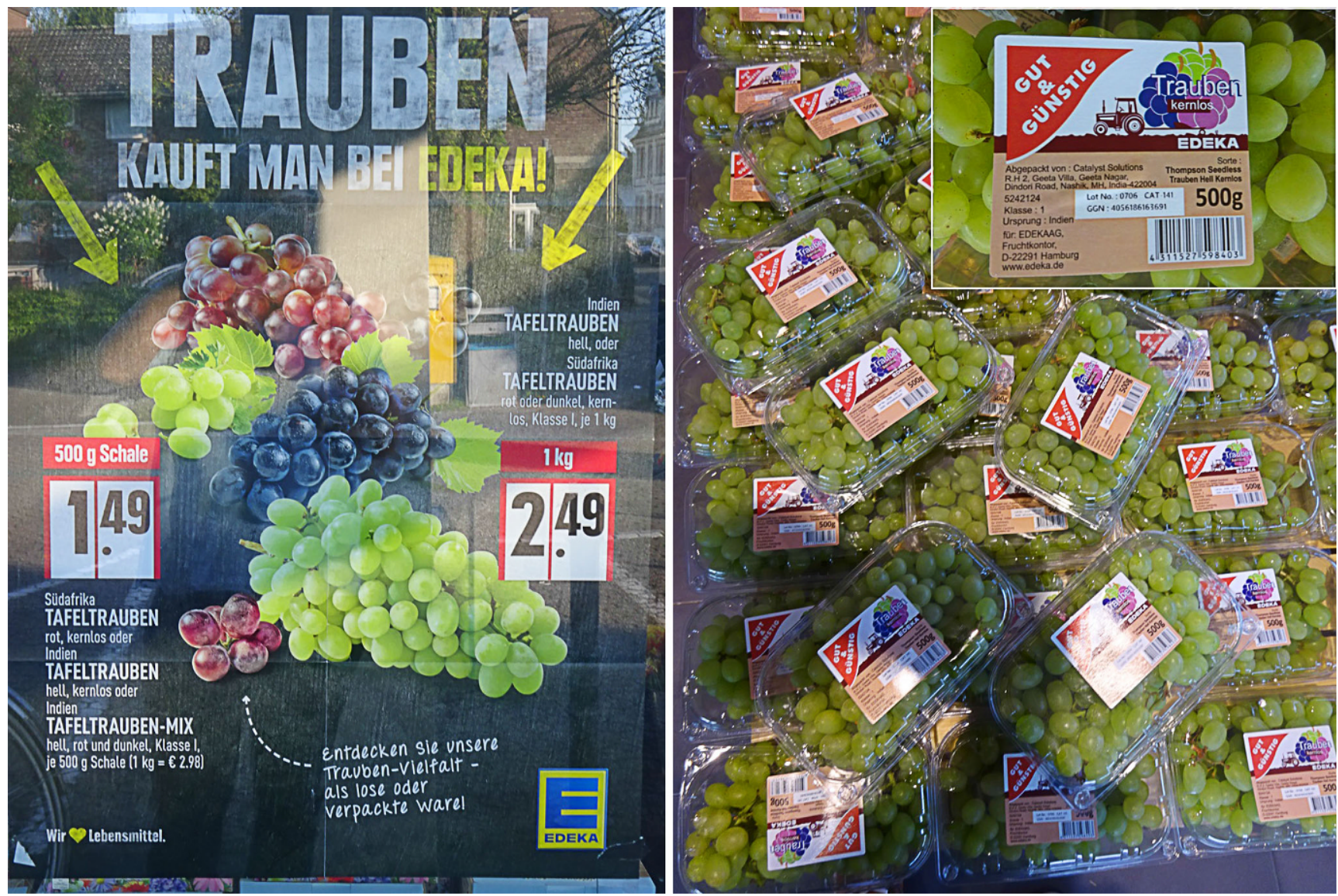

Abb. 4 Indische 'Thompson Seedless'-Trauben im April 2019 im deutschen LEH

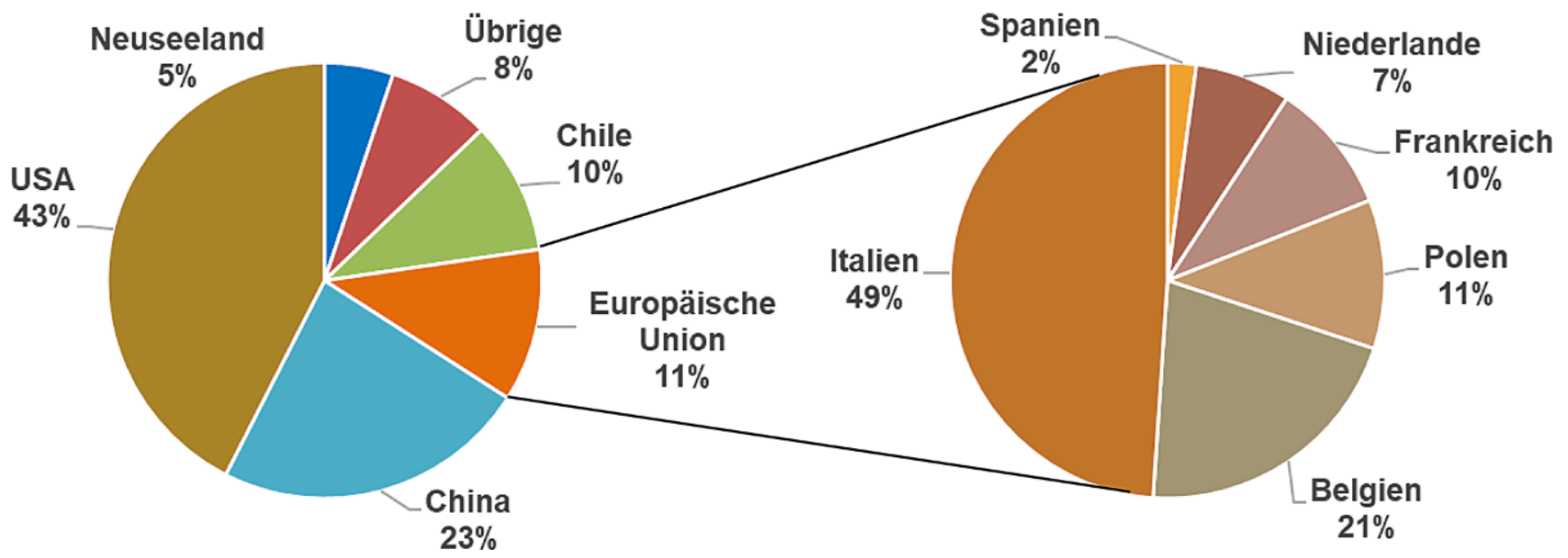

Abb. 5 Anteile Indiens Apfelimportländer von April 2017-März 2018 (Bezugsbasis der Statistik ist der Warenwert in US-Dollar Ministry of Commerce und Industry 2019)

und Fäulnis aussortiert und die Ware neu - wiederum in Holzkisten - verpackt (Abb. 6) und ansprechend angeboten (Abb. 7), die Holzkisten werden nachhaltig wie in einem Kistenpool wiederverwendet. Beschädigte, aussortierte Äpfel werden z.T. als Mostobst der Saftindustrie für $20 \%$ des ursprünglichen Preises verkauft oder stehen bedürftigen
Menschen kostenlos zur Verfügung, d.h. nur ein Teil der beschädigten Äpfel geht verloren.

Verluste können auch bei unzureichender Lagerung in einfachen Schuppen entstehen (Kireeti und Sharma 2017; Melendez et al. 2017). Clevere Anbauer nutzen nachts die kalte Luft aus den Bergen und öffnen dann ihre Lagerräu- 


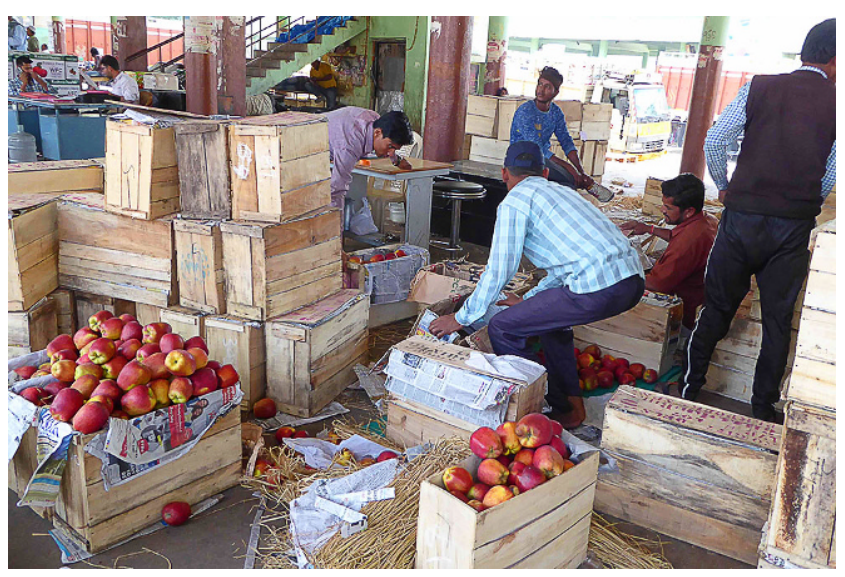

Abb. 6 Umpacken indischer Äpfel von Holzkisten in Holzkisten

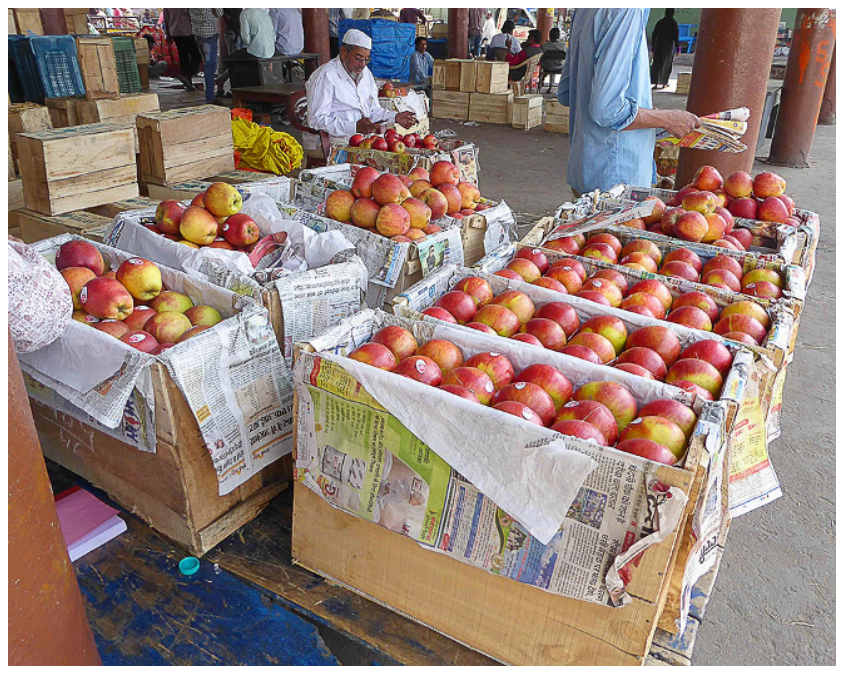

Abb. 7 Präsentation indischer Äpfel auf einem Obstmarkt bei Hyderabad - Nachhaltige Verpackung ohne Plastik

me (Wani et al. 2018). Weitere Kühl- und CA-Lager werden errichtet, um Lagerverluste zu vermindern und um Äpfel später, und dann gewinnbringender, verkaufen zu können. Bisher besteht noch ein akuter Mangel an bezahlbaren Kühllagern auf allen Ebenen der Vermarktungskette. Der indische Staat fördert die Errichtung von weiteren Kühlund CA-Lagern. Inzwischen bieten indische Firmen auch solarbetriebene Kühllager vieler Größen an (z.B. ecoZen, ecofrost). Mit den zukünftigen Kühlkapazitäten sollen auch die angestrebten Ertragssteigerungen besser genutzt werden.

Dagegen werden die ausländischen Äpfel in Kühltransportern von Häfen wie Mumbai (ehemals Bombay), Kolkata (Kalkutta) (Abb. 1) und Chennai (Madras) zu den Obstmärkten wie Hyderabad mit fast geschlossener Kühlkette geliefert.

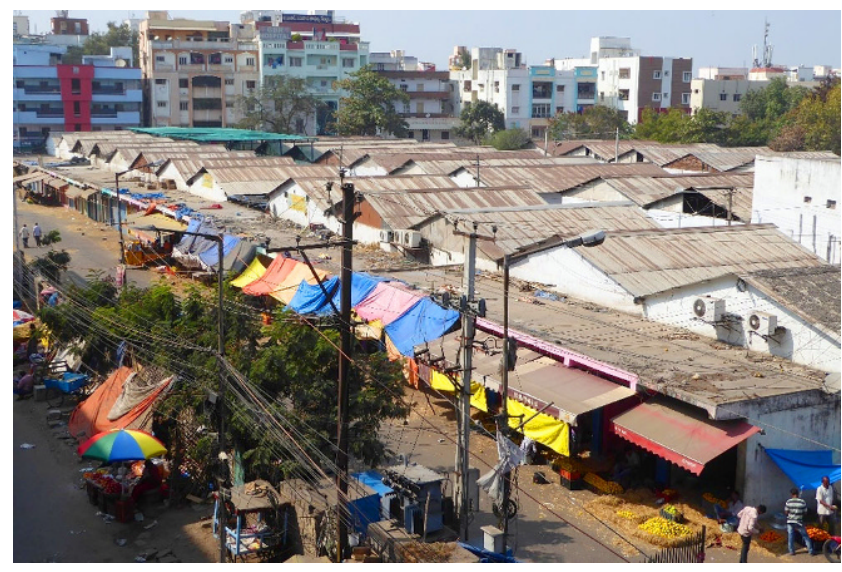

Abb. 8 Der Kothapet Obstgroßmarkt in Hyderabad mit der zentralen Vermarktungshalle gesäumt von kleinen Obsthändlern

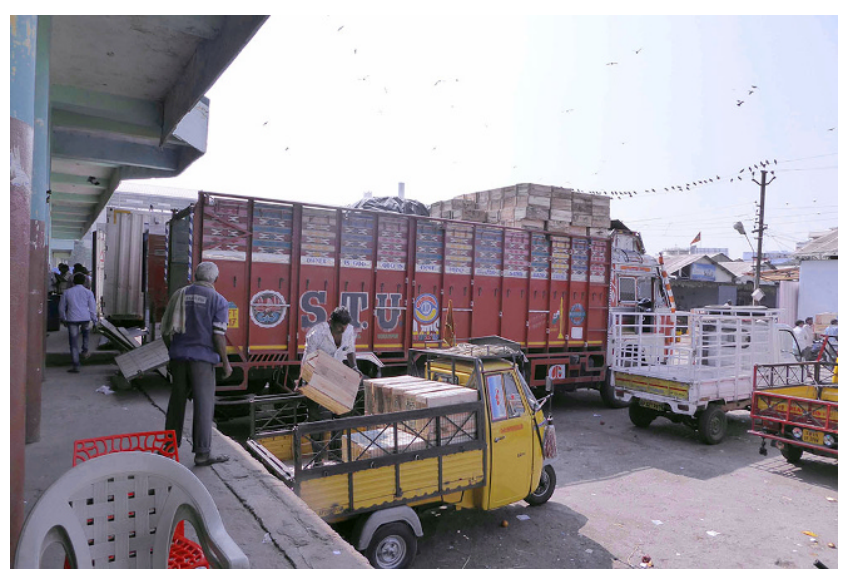

Abb. 9 Verladerampe - Anlieferung und Abholung indischer Äpfel ohne Kühltransporte

\section{Vermarktung auf dem Obstmarkt Kothapet/ Hyderabad und in Hyderabad}

Äpfel werden von den Großhändlern entweder in einer Auktion oder in Kommission mit einem ,commission agent“ mit $5 \%$ Gewinnanteil verkauft (Abb. 8 und 9). Straßenverkäufer tragen die auf dem Großmarkt (Abb. 10) erworbenen Kartons zu ihrem Stand (Abb. 11) oder nutzen Verkaufskarren (Abb. 12) und verkaufen nach Gewicht oder nach Stück, je nachdem, ob sie eine Waage haben. Diese Art des Obstverkaufens ist eine Möglichkeit, Geld ohne Anstellung und Ausbildung zu verdienen. Weitere Einkäufer beim Großmarkt sind Obstgeschäfte und Supermärkte. 


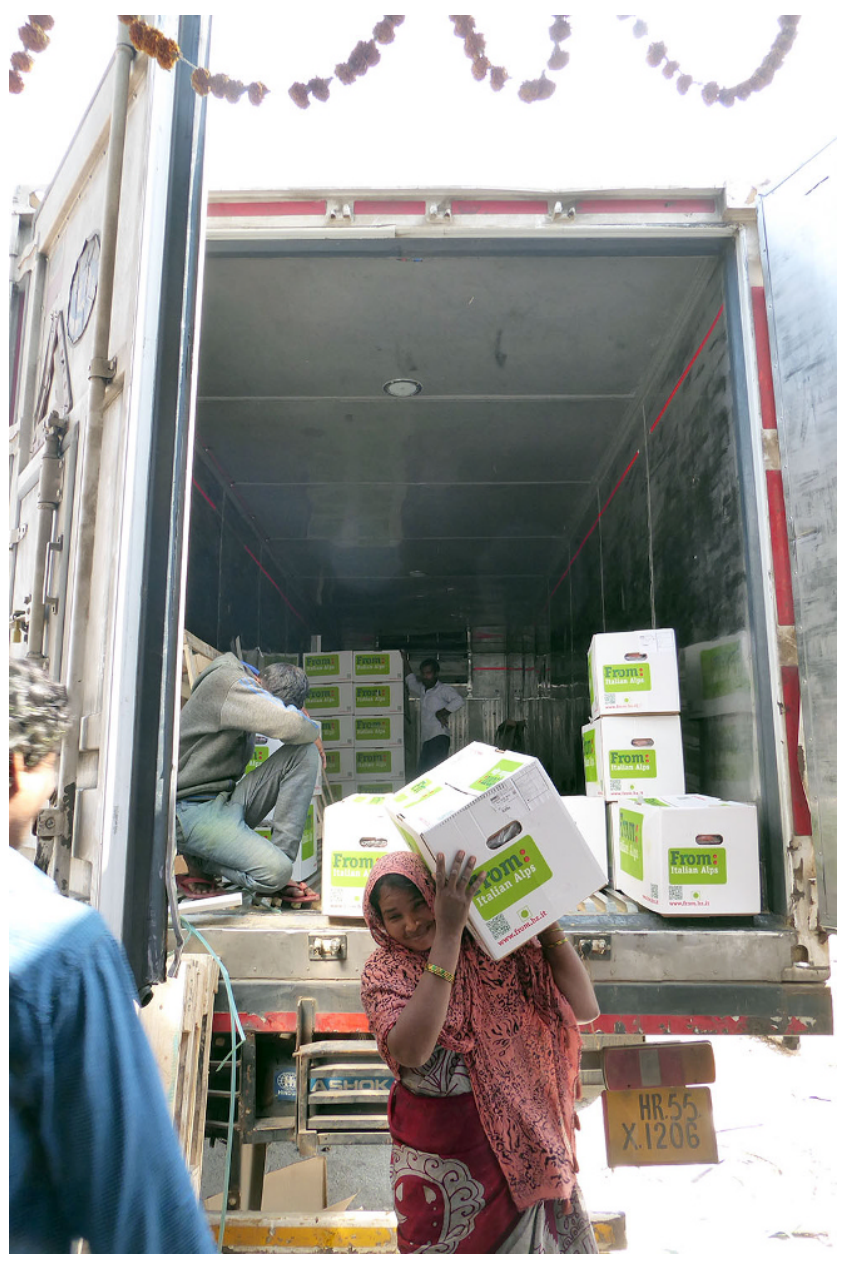

Abb. 10 Ausladen italienischer Äpfel aus Südtirol aus dem Kühltransporter von Hand

\section{Konsumpräferenzen auf dem Indischen Markt und Preise in Abhängigkeit von Fruchtgröße, Rotfärbung, Herkunft und Marktform}

Der indische Konsument bzw. Markt präferiert große (>75 mm), rote (oder grüne) Äpfel ohne Schalenfehler bei geringem Marktanteil grüner Äpfel. Die Konsumpräferenzen spiegeln sich z.B. in den Stückpreisen indischer 'Red Delicious'-Äpfel von 10 bis $25 \mathrm{Rs}$ - also Faktor 2,5 - und in den indischen Qualitätsklassen A-C (Ahmad et al. 2014) wider, wobei A 70-100\%, B 50-70\% und C 0-100\% roter Schalenanteil beschreibt.

Die großen Äpfel können dabei eher eine Folge geringen Fruchtbehanges - als Folge unzureichenden Kältereizes und Blüte oder gezielter Ausdünnungsmaßnahmen sein.

Im Januar 2019 waren die Endpreise der Äpfel aus Kaschmir und Himachal Pradesh vergleichbar mit denen anderer Früchte. Die ausländischen Äpfel (Abb. 13) waren fast doppelt so teuer und eben „trendy“, weil man - wie

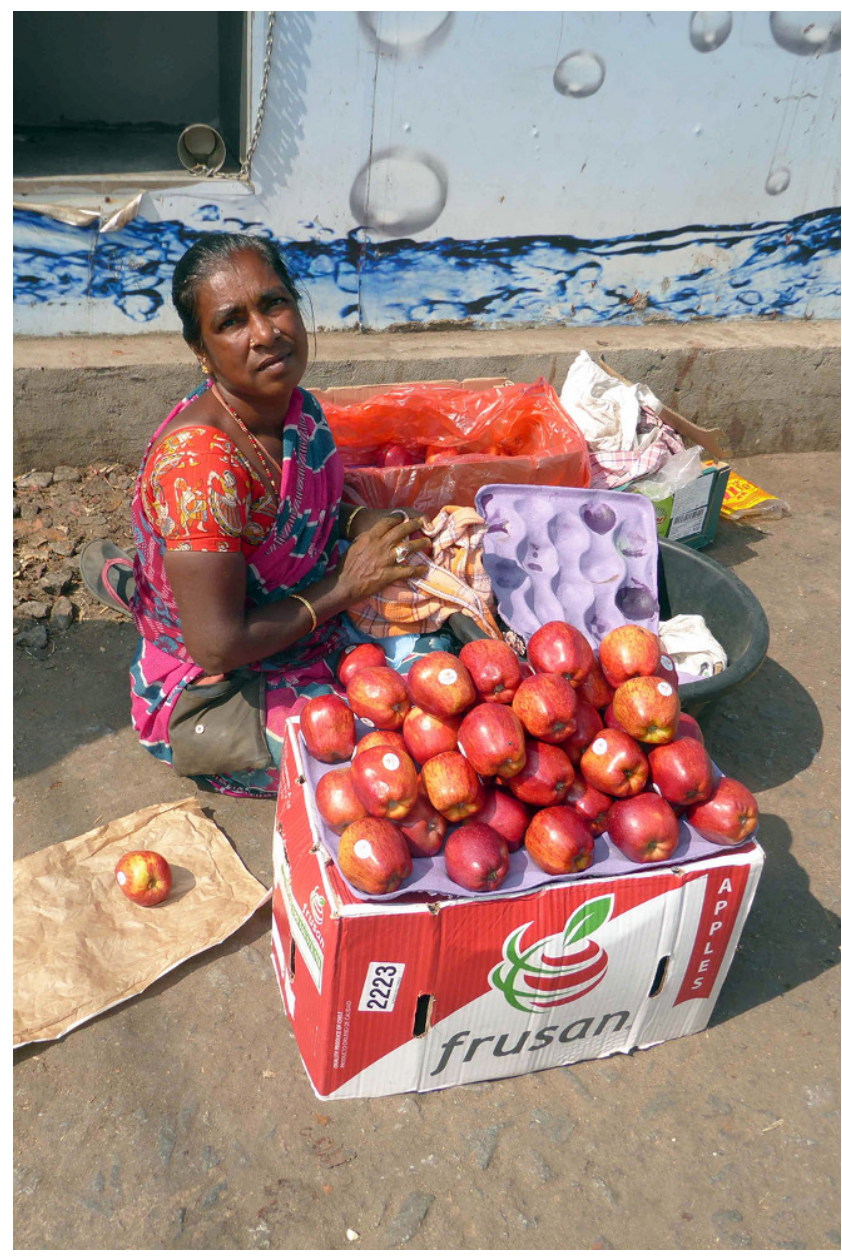

Abb. 11 Straßenverkäuferin mit Red Delicious aus Chile

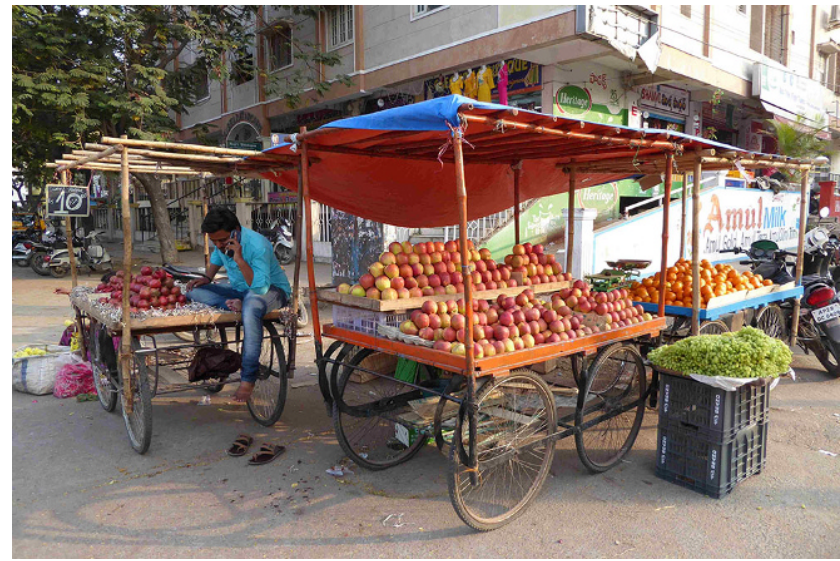

Abb. 12 Verkaufskarren am Straßenrand für Äpfel, Orangen und Granatäpfel

früher in Russland - annahm, alles aus dem Ausland sei erstmal besser (Blanke 2020).

Während in zwei Supermärkten (,organised markets“) mit wohlsituierten Kunden das Angebot ausländischer Äpfel im Januar überwog, kehrte sich das Verhältnis auf den 


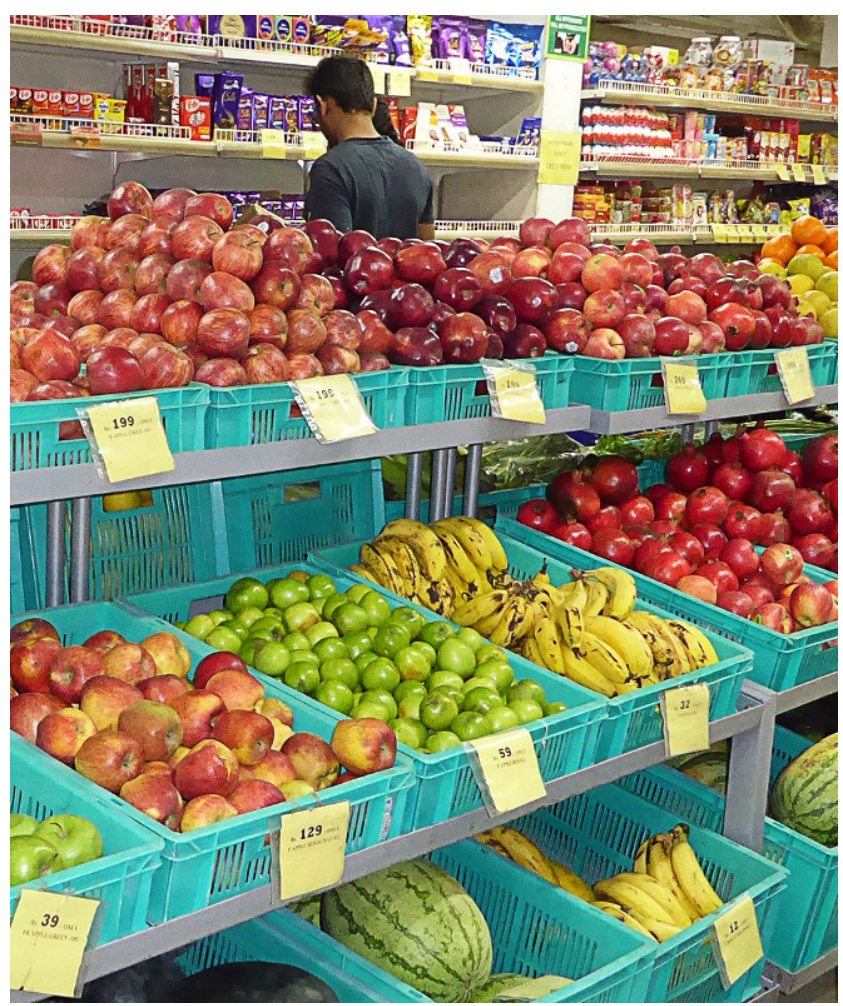

Abb. 13 Sorten und Preise in einem Supermarkt in Indien: v. 1. n. r.: obere Reihe: Fuji (USA) zu Rs 229, zwei rote Sorten (Chile) zu Rs 199, 'Red Delicious‘ (USA), 'Rosy Glow' (Italien) und Granatäpfel (Rs 199); untere Reihe: 'Granny Smith' (Preis pro Stück/Apfel Rs 39), indische Äpfel aus Himachal Pradesh (Rs 129)
Tab. 2 Vergleich der Apfel- bzw. Sortenherkunft im Angebot

\begin{tabular}{lll}
\hline Herkunft Inland & $\begin{array}{l}\text { Sorten aus dem } \\
\text { Ausland }\end{array}$ & Herkunft Ausland \\
\hline $\begin{array}{l}\text { 'Red Delicious' aus } \\
\text { Kaschmir } \\
\text { 'Maharaji' aus H. Pra- } \\
\text { desh }\end{array}$ & 'Red Delicious' & Chile USA \\
- & 'Royal Gala' & $\begin{array}{l}\text { Italien Polen Neu- } \\
\text { seeland }\end{array}$ \\
- & Granny Smith & Italien Neuseeland \\
- & 'Fuji' & USA \\
\hline & 'Pacific Rose' & Neuseeland \\
\hline
\end{tabular}

Tab. 3 Preise in Hyderabad Januar 2019 (bei Rs= indische Rupien, $1 €=82 \mathrm{Rs})$

\begin{tabular}{lll}
\hline Herkunft & Großmarkt Hyderabad & Einzelhandel Hyderabad \\
\hline Inländisch & $28-35 \mathrm{Rs} / \mathrm{kg}$ & $0,80-129 \mathrm{Rs} / \mathrm{kg}$ \\
& $0,34-0,43 € / \mathrm{kg}$ & $0,98-1,57 € / \mathrm{kg}$ \\
Ausländisch & $56-145 \mathrm{Rs} / \mathrm{kg}$ & $199-300 \mathrm{Rs} / \mathrm{kg}$ \\
& $0,69-1,77 € / \mathrm{kg}$ & $2,43-3,66 € / \mathrm{kg}$ \\
\hline
\end{tabular}

Straßenmärkten um. Sowohl im Supermarkt als auch auf dem Straßenmarkt wird ein teurer 'green apple' verkauft, bei dem es sich häufig um importieren 'Granny Smith' aus Neuseeland, Chile oder Südtirol (Tab. 2) handeln dürfte (Yuri und Blanke 2020). Der Großteil der Äpfel wird über Straßenmärkte verkauft.
Abb. 14 Dekorierte Äpfel als Symbol bzw. Wunsch für Wohlstand als Hochzeitsgeschenk (C) Kavitha Ramesh - mit Genehmigung)
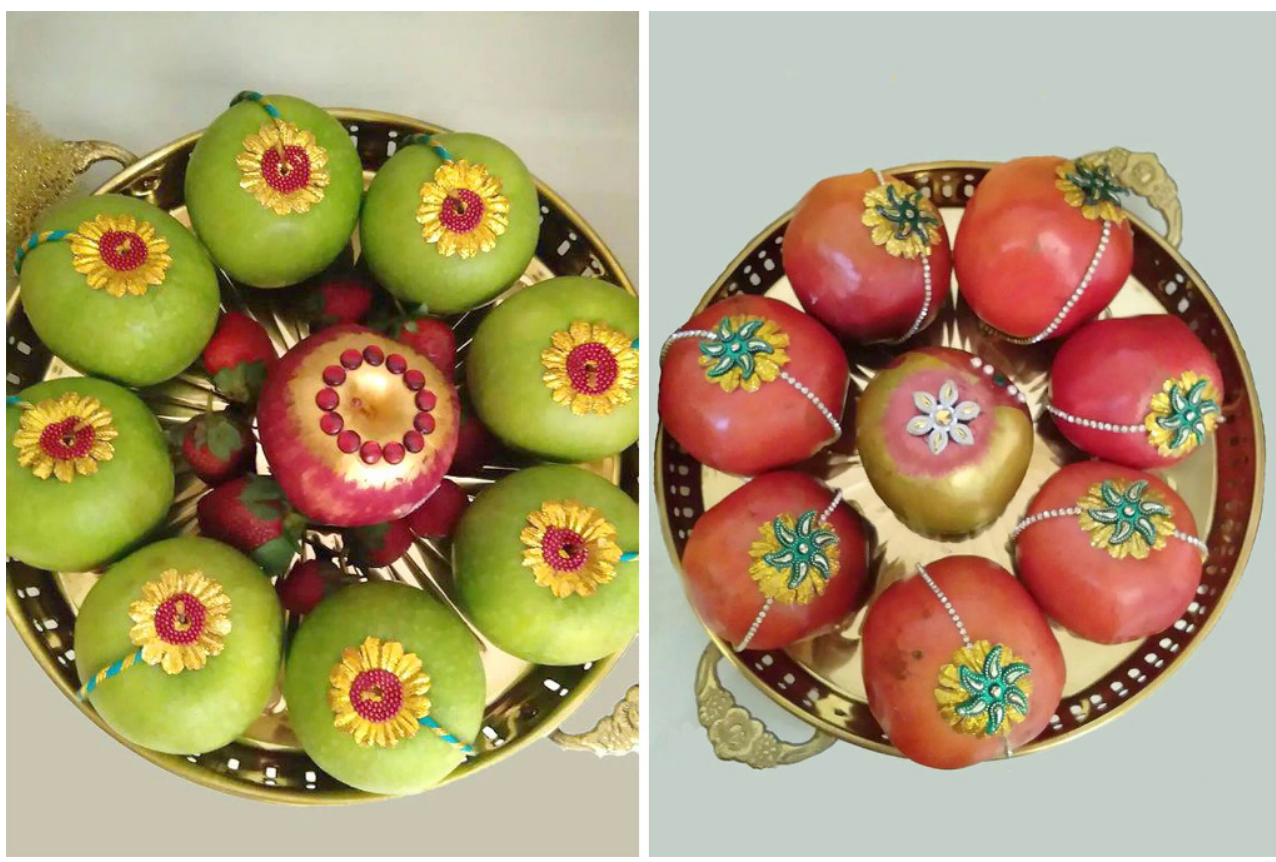
Abb. 15 Wertschöpfungskette Äpfel in Indien mit dem Flaschenhals Einzelhändler am Großmarkt
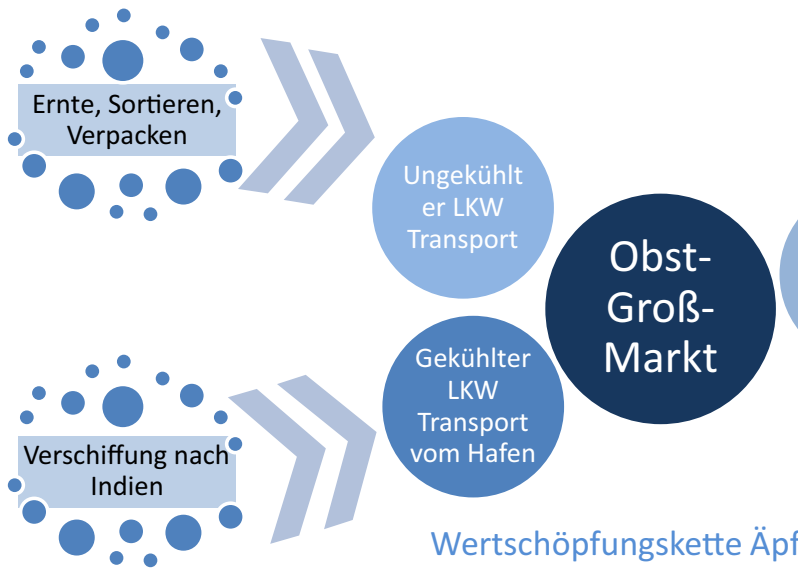

Wertschöpfungskette Äpfel in Indien

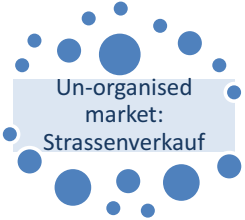

Entladen/

Transport

von Hand/

Moped
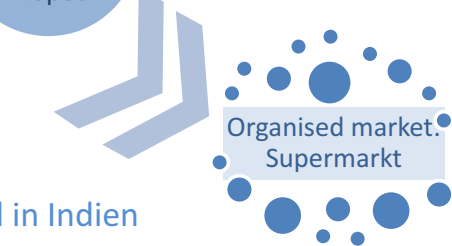

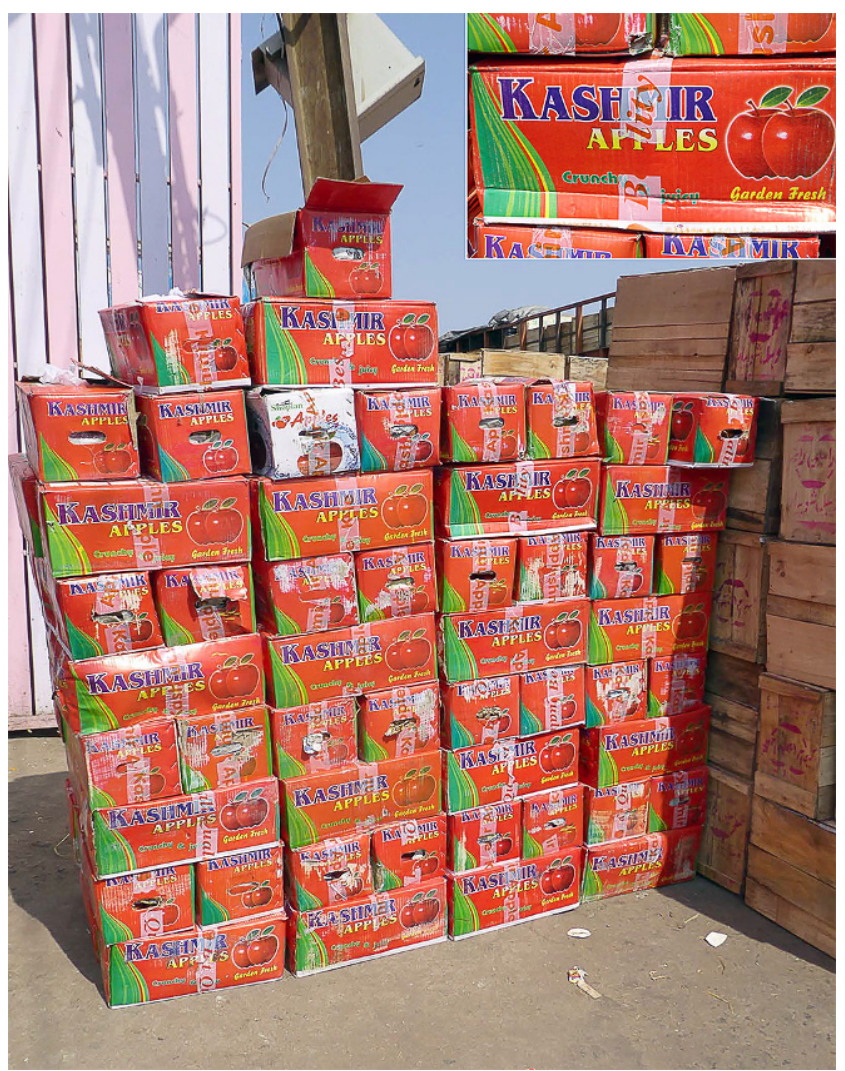

Abb. 16 Äpfel aus der Grenzregion Kaschmir - in Kartonage verpackt

\section{Apfel - Zeichen des Wohlstandes und Opfer in der Religion}

Auch wenn die Mango in Indien als die Königin aller Früchte angesehen wird, besitzt der Apfel Symbolcharakter für Wohlstand - als Geschenk der Brauteltern an die Familie des Bräutigams (Abb. 14) und als Darbietung nach der Andacht im Hindutempel als Prasad für die Gläubigen. Äpfel als religiöse Opfergaben stehen den Priestern oder Gläubi- gen anschließend zum Verzehr zur Verfügung, d.h. führen nicht zu Lebensmittelabfällen.

\section{Preisvergleich heimischer, indischer Äpfel versus hohe Preise für Importware}

Auf dem Großmarkt in Hyderabad lagen die Preise im Januar 2019 bei den Großhändlern für inländische Äpfel bei 28-35Rs/kg (0,34-0,43€/kg) und für ausländische Äpfel bei $56-145 \mathrm{Rs} / \mathrm{kg}(0,69-1,77 € / \mathrm{kg})$ (Tab. 3), wobei auch Zwischenhändler auf diesem Großmarkt einkaufen und dann wieder teurer an die Endhändler verkaufen (Abb. 15). Die Gewinnspanne zwischen Großmarkt und LEH beträgt $1 / 2$ bis $2 / 3$ des Endpreises, d.h. bei einem Preis von $€ 1,5$ zwischen $€ 0,75$ bis $€ 1 / \mathrm{kg}$.

\section{Umstrittene Apfelherkunft auf dem Straßenmarkt in Hyderabad - Neues Apfelanbaugebiet in Telangana und Saisonalität}

Im Januar wiesen die Straßenverkäufer in Hyderabad bei den nordindischen Äpfeln auf ihre Herkunft hin, die zu dieser Zeit aus dem entfernteren Grenzgebiet Kaschmir (ca. $2500 \mathrm{~km}$; Abb. 16) - und nicht aus Himachal Pradesh (ca. $2000 \mathrm{~km})$ - stammen. Zukünftig werden wohl auch Äpfel aus dem Norden von Telangana, dem Bundesstaat von Hyderabad, nach Hyderabad geliefert; dort entstehen in einer höhergelegenen Region neue Apfelplantagen. Wenn in Hyderabad ab März weniger Äpfel aus Nordindien zur Verfügung stehen, werden in geringem Umfang ausländische Äpfel angeboten, die den meisten Apfelkäufern zu teuer sind. In wärmeren Süden Indiens wachsen auch viele (sub-)tropische Früchte, so dass Äpfel bei geringer Verfügbarkeit, Geschmackseinbußen bei Qualitätsverlusten 
und höheren Preisen bei guten Qualitäten weniger häufig gegessen werden als im kühleren Norden Indiens.

\section{Fruchtqualität und Geschmacksproben Januar 2019}

Bei einer Verköstigung im Januar schmeckten die 'Red Delicious' Äpfel aus Nordindien überlagert mit wenig Süße und wenig Säure, weich, nicht saftig; einzelne Früchte zeigten Druckstellen und wiesen einen unangenehmen Beigeschmack auf. Für die Käufer, die sich die teuren Äpfel nicht leisten können, ist diese Qualität ausreichend, denn diese Äpfel werden verkauft.

Die ausländischen fast doppelt so teuren Äpfel wiesen das ganze Spektrum von wenig bis sehr schmackhaft bezüglich Süße, Säure, Festigkeit und Saftigkeit mit weniger Druckstellen und ohne unangenehmen Beigeschmack auf.

\section{Fazit}

Die riesigen Apfel-Potentiale Indiens liegen in einer vielfachen Steigerung des Flächenertrages, Verringerung der hohen Lager- und Transportverluste und Importpotenzial von 250.000 bis $400.000 t$ jährlich bei hohen Preisen und zunehmender Nachfrage nach gesundem Obst und Gemüse Dank steigendem Wohlstand, Gesundheits- und Umweltbewusstsein bzw. auch staatlichen Bemühungen um einen weitgehenden Verzicht auf Einwegplastik, so dass zur Zeit viele Entwicklungsprojekte des indischen Staates - auch in Zusammenarbeit mit der Weltbank - durchgeführt werden.

Danksagung Die Autoren bedanken sich bei Kavita Ramesh für die Abdruckgenehmigung der beiden Fotos mit den dekorierten Äpfeln als Hochzeitsgeschenk der Brauteltern an den Bräutigam.

Funding Open Access funding provided by Projekt DEAL.

Interessenkonflikt S. Golombek und M. Blanke geben an, dass kein Interessenkonflikt besteht.

Open Access Dieser Artikel wird unter der Creative Commons Namensnennung 4.0 International Lizenz veröffentlicht, welche die Nutzung, Vervielfältigung, Bearbeitung, Verbreitung und Wiedergabe in jeglichem Medium und Format erlaubt, sofern Sie den/die ursprünglichen Autor(en) und die Quelle ordnungsgemäß nennen, einen Link zur Creative Commons Lizenz beifügen und angeben, ob Änderungen vorgenommen wurden.

Die in diesem Artikel enthaltenen Bilder und sonstiges Drittmaterial unterliegen ebenfalls der genannten Creative Commons Lizenz, sofern sich aus der Abbildungslegende nichts anderes ergibt. Sofern das betreffende Material nicht unter der genannten Creative Commons Lizenz steht und die betreffende Handlung nicht nach gesetzlichen Vorschriften erlaubt ist, ist für die oben aufgeführten Weiterverwendungen des Materials die Einwilligung des jeweiligen Rechteinhabers einzuholen.
Weitere Details zur Lizenz entnehmen Sie bitte der Lizenzinformation auf http://creativecommons.org/licenses/by/4.0/deed.de.

\section{Literatur}

Ahmad MS, Nayyer MA, Aftab A, Nayak B, Siddiqui MW (2014) Quality prerequisites of fruits for storage and marketing. J Postharvest Technol 2:107-123

Arora T (2018) Potential for apple importers. INTERPOMA, 15. Nov. 2018 (Podiumsvortrag)

Blanke MM (2020) Apfelmarkt in Russland. Erwerbs-Obstbau 62(4). (im Druck)

Chauhan K (2016) Cold desert apple orcharding. Fruit Notes 81:1-6

FAO (2018) Crops statistics for 2017. http://www.fao.org/faostat/en/\# data/QC. Zugegriffen: 06.01.2019

Golombek S, Blanke MM (2020) $\mathrm{CO}_{2}$-Fußabdruck indischer Tafeltrauben auf dem Weg zum deutschen Konsumenten - Analyse und Ansätze zu seiner Verminderung. Erwerbs-Obstbau. https://doi. org/10.1007/s10341-020-00514-w

Horticulture Statistics Division, Department of Agriculture, Cooperation \& Farmers Welfare, Ministry of Agriculture \& Farmers Welfare, Government of India (2017) Horticultural statistics at a glance 2017. http://nhb.gov.in/statistics/Publication/ Horticulture\%20At\%20a\%20Glance\%202017\%20for\%20net \%20uplod\%20(2).pdf. Zugegriffen: 22.02.2018

Khan JN, Dixit J, Kumar R (2018) Mechanization options for apple based production system in India. In: Ahmed N, Wani SA, Wani WM (Hrsg) Apple. Production and value chain analysis, 1. Aufl. Daya Pub. House, New Delhi, S 327-336

Kireeti K, Sharma LR (2017) An inquiry into the problems of apple production and marketing in the perspective of apple growers in Shimla district of Himachal Pradesh. Int J Agric Sci Res 7:7-14. https://doi.org/10.24247/ijasraug20172

Melendez P, Massa V, Shah K, Muller J, Kalal N, Jain M (2017) Extending the apple season: cold storage in Himachal Pradesh, India. https://web.wpi.edu/Pubs/E-project/Available/E-project-050217070024/unrestricted/IN17-FOOD_FinalReport.pdf. Zugegriffen: 22. Febr. 2019

Ministry of Commerce and Industry (2019) Export import data bank, Department of Commerce, Ministry of Commerce \& Industry, Government of India. http://commerce-app.gov.in/eidb/ icomxcntq.asp. Zugegriffen: 06.01.2019

Singh J, Patel NR (2017) Assessment of agroclimatic suitability of apple orchards in Himachal Pradesh under changing climate. J Agrometeorol 19:110-113

Terlau W, Hirsch D, Blanke MM (2018) Smallholder farmers as the backbone of the implementation of the sustainable development goals (SDGs). Sustain Dev 26(6):1000-1004. https://doi.org/10. 1002/sd.1907

Wani SA, Naqash F, Shaheen FA, Manzoor H (2018) CA storage of apple. In: Ahmed N, Wani SA, Wani WM (Hrsg) Apple. Production and value chain analysis, 1. Aufl. Daya Pub. House, New Delhi, S $411-420$

Weltbank (2018) India. https://data.worldbank.org/country/india. Zugegriffen: 18. März 2019

Werth K, Kager J (2018) Die Apfelanbaugebiete in Osteuropa. INTERPOMA, Bozen, 15. Nov. 2018 (Vortrag)

Yuri A, Blanke MM (2020) Chile - Exportrekorde im Obstbau vom Fuße der Anden. Erwerbs-Obstbau 62:175-180. https://doi.org/ 10.1007/s10341-020-00474-1 


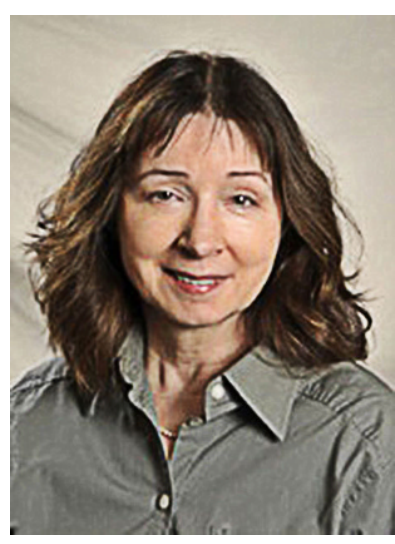

Sabine Golombek Dr., studierte Agrarbiologie an der Universität Stuttgart-Hohenheim. Nach ihrer Promotion 1991 am Obstbauinstitut bei Prof. P. Lüdders über die Physiologie subtropischer Obstkulturen verbrachte sie einen Post-Doc am ICRISAT in Hyderabad in Indien und hält bis heute den Kontakt zu den dortigen Wissenschaftlern und Land und Kultur mit regelmäßigen Besuchen. Danach beschäftigte sie sich am IPK in Gatersleben mit dem Proteinstoffwechsel von Leguminosen und an der Hochschule Kassel/Witzenhausen mit angewandten Fragestellungen von Nutzpflanzen. Sie verfügt über Erfahrungen in Lehre (Obst- und Pflanzenbau), Forschung mit entsprechenden Publikationen und Forschungsanträgen. Ihr Interesse gilt außer den heimischen und subtropischen Obstgehölzen allgemein dem Anbau, der Verwertung und Physiologie der Nutzpflanzen. 